\title{
Obstructive sleep apnea-hypopnea syndrome (OSAHS) comorbid with diabetes rather than OSAHS alone serves an independent risk factor for chronic kidney disease (CKD)
}

\author{
Miao Hui ${ }^{1}$, Yuehong Li $^{1}$, Jingying Ye ${ }^{2}$, Zhen Zhuang ${ }^{1}$, Wei Wang ${ }^{1}$ \\ ${ }^{1}$ Department of Nephrology, ${ }^{2}$ Department of Otolaryngology, Beijing Tsinghua Changgung Hospital, School of Clinical Medicine, Tsinghua \\ University, Beijing 102218, China \\ Contributions: (I) Conception and design: M Hui, Y Li, J Ye; (II) Administrative support: Y Li; (III) Provision of study materials or patients: All \\ authors; (IV) Collection and assembly of data: All authors; (V) Data analysis and interpretation: M Hui; (VI) Manuscript writing: All authors; (VII) \\ Final approval of manuscript: All authors. \\ Correspondence to: Yuehong Li. Department of Nephrology, Beijing Tsinghua Changgung Hospital, School of Clinical Medicine, Tsinghua University, \\ Beijing 102218, China. Email: liyuehong0616@163.com; Jingying Ye. Department of Otolaryngology, Beijing Tsinghua Changgung Hospital, School \\ of Clinical Medicine, Tsinghua University, Beijing 102218, China. Email: yejingying@vip.163.com.
}

\begin{abstract}
Background: This retrospective study aimed to investigate the effect of metabolic diseases on the relationship between obstructive sleep apnea-hypopnea syndrome (OSAHS) and chronic kidney disease (CKD) and explore the contributing factors.

Methods: A total of 424 adult patients with OSAHS were included, and 20 patients with simple snoring syndrome served as controls. Estimated glomerular filtration rate (eGFR) $<60 \mathrm{~mL} / \mathrm{min} / 1.73 \mathrm{~m}^{2}$ or proteinuria was diagnosed as CKD. The prevalence of CKD and the correlation between OSAHS comorbid with metabolic diseases and CKD were analyzed.

Results: The prevalence of CKD in OSAHS patients was $25.5 \%$, which was 5.1 times higher than that in patients with simple snoring syndrome (5.0\%). The prevalence of CKD in OSAHS patients comorbid with metabolic diseases was 1.9 times higher than that in OSAHS patients without metabolic diseases $(28.5 \% v s$. $15.3 \%, \mathrm{P}<0.05)$. OSAHS comorbid with diabetes rather than OSAHS alone was an independent risk factor for $\mathrm{CKD}[\mathrm{P}=0.045$, odds ratio $(\mathrm{OR})=0.566,95 \%$ confidence interval $(\mathrm{CI}): 0.324-0.989]$. In OSAHS patients with diabetes, the prevalence of CKD comorbid with 1 to 4 metabolic diseases was $25.0 \%, 34.4 \%, 43.2 \%$ and $66.7 \%$, respectively. With the increase in the number of comorbidities of metabolic diseases, the prevalence of CKD gradually increased.

Conclusions: The prevalence of CKD in OSAHS patients is relatively high. OSAHS comorbid with diabetes rather than OSAHS alone is an independent risk factor for CKD. The prevalence of CKD increases with the number of comorbidities of metabolic diseases.
\end{abstract}

Keywords: Obstructive sleep apnea (OSA); metabolic disease; chronic kidney disease (CKD)

Submitted Oct 02, 2019. Accepted for publication Feb 12, 2020.

doi: 10.21037/apm.2020.03.21

View this article at: http://dx.doi.org/10.21037/apm.2020.03.21

\section{Introduction}

Obstructive sleep apnea-hypopnea syndrome (OSAHS) is a common sleep disorder that is characterized by obstructive apneas, hypopneas, and/or respiratory effort-related arousals caused by repetitive collapse of the upper airway during sleep (1). OSAHS is associated with numerous risk factors, including race, age, male, obesity, menopause craniofacial structural abnormalities and adverse living habits, such as smoking and drinking (2-5). Patients with OSAHS also have an increased prevalence of other 
diseases, such as hypertension, cardiovascular disease (6), type 2 diabetes mellitus (T2DM). However, it is often ignored by physicians and patients. In clinical practice, $82 \%$ of male patients and $93 \%$ of female patients with moderate to severe sleep apnea have not been diagnosed (7). Due to the increasing prevalence of overweight and obesity, more attention has been paid to OSAHS. CKD is defined by the presence of kidney damage or decreased kidney function lasting for $>3$ months, with implications for health (8). CKD has been a common disease seriously threatening human health in addition to cardiovascular and cerebrovascular diseases, diabetes and malignant tumors, and has become the 11th leading cause of death by 2016 (9). An increasing prevalence of CKD has been reported in the OSAHS patients (7). As an organ rich in blood perfusion, kidney function is sensitive to change in oxygen supply and susceptible to hypoxia injury. Although the correlation between OSAHA and CKD has been reported, but there are selective biases in available studies, such as diagnostic methods, exclusion factors, sample size, sample representativeness and comorbidity. Thus, the correlation between OSAHS and CKD and the effect of OSAHS on CKD should be further elucidated.

\section{Methods}

Totally 2,292 patients over 18 years old who completed whole night polysomnography (PSG) in Beijing Tsinghua Changgung Hospital from Dec 2014 to Nov 2018 were reviewed retrospectively. Of them, 424 patients were diagnosed with OSAHS and 20 patients with simple snoring. OSAHS was classified as mild, moderate and severe according to the Apnea-Hypopnea Index (AHI) (5-15, >15-30 and $>30)$. Subjects received PSG monitoring for at least 7 consecutive hours during sleep at night. All patients before sleep monitoring did not take sedatives or hypnotizers, and not take food or drugs containing alcohol or caffeine. PSG monitoring involved oral and nasal airflow, chest and abdomen movement, AHI, blood oxygen saturation, and oxygen desaturation index (ODI). Simple snoring (control group) was considered when AHI was $<5$ times/h. OSAHS was diagnosed when AHI was $\geq 5$ times/h. All patients were not treated for any sleep apnea disorder.

Central and mixed sleep apnea hypopnea syndrome and other sleep-related diseases, CKD of known causes, chronic obstructive pulmonary disease (COPD) and asthma, autoimmune diseases, cirrhosis or malignant tumors, recent urinary system infection, nephrotoxic medication were excluded from those patients.

\section{Data collection}

General information such as age, gender, history of obesity, diabetes, hypertension, hyperlipidemia and hyperuricemia, blood pressure (systolic/diastolic), height and weight. AHI, lowest saturated carbon saturation $\left(\mathrm{LSaO}_{2}\right)$, mean blood oxygen saturation $\left(\mathrm{MSaO}_{2}\right)$ and ODI was recorded. ODI was defined as the value of desaturation of blood oxygen per hour. Blood oxygen desaturation referred to the decrease in oxygen saturation is greater than $4 \%$, which lasted from 10 seconds to 3 minutes).

Detections of serum creatinine, urea nitrogen, liver function, lipids, uric acid, fasting blood glucose (FBG), glycaemia hemoglobin and urine routine test (qualitative/quantitative urine protein) were done. CKD was defined as estimated glomerular filtration rate $(\mathrm{eGFR}) \leq 60 \mathrm{~mL} / \mathrm{min} / 1.73 \mathrm{~m}^{2}$ for 3 months calculated with the CKD-Epidemiology Collaboration (EPI) creatinine formula.

\section{Statistical analysis}

Statistical analysis was performed with SPSS version 25.0 for MAC (SPSS Inc., Chicago, IL, USA). Continuous variables with normal distribution were expressed as mean \pm standard deviation (SD) and compared with $t$-test. The ratios were compared with Chi-square test. Pearson correlation analysis was used to analyze the correlation among variables. Multivariate linear regression and binary Logistic regression analyses were used to explore the independent risk factors. A value of $\mathrm{P}<0.05$ was considered statistically significant.

\section{Results}

\section{Clinical baseline characteristics}

Four hundred and twenty-four patients were diagnosed with OSAHS, and 20 patients with simple snoring served as the control group. The clinical baseline characteristics are shown in Table 1. In the OSAHS group, there were 343 males $(80.9 \%)$ and 81 females $(19.1 \%)$ with an average age of $47.8 \pm 13.2$ years. There were 108 patients $(25.5 \%)$ with CKD, with a mean eGFR of $103.5 \pm 20.5 \mathrm{~mL} / \mathrm{min} / 1.73 \mathrm{~m}^{2}$, and 93 patients $(21.9 \%)$ had positive urinary protein. The prevalence of CKD in the control group was $5.0 \%$, with a mean eGFR of $104.9 \pm 20.4 \mathrm{~mL} / \mathrm{min} / 1.73 \mathrm{~m}^{2}$. The prevalence 
Table 1 Clinical characteristics of patients in the OSAHS group and simple snoring group

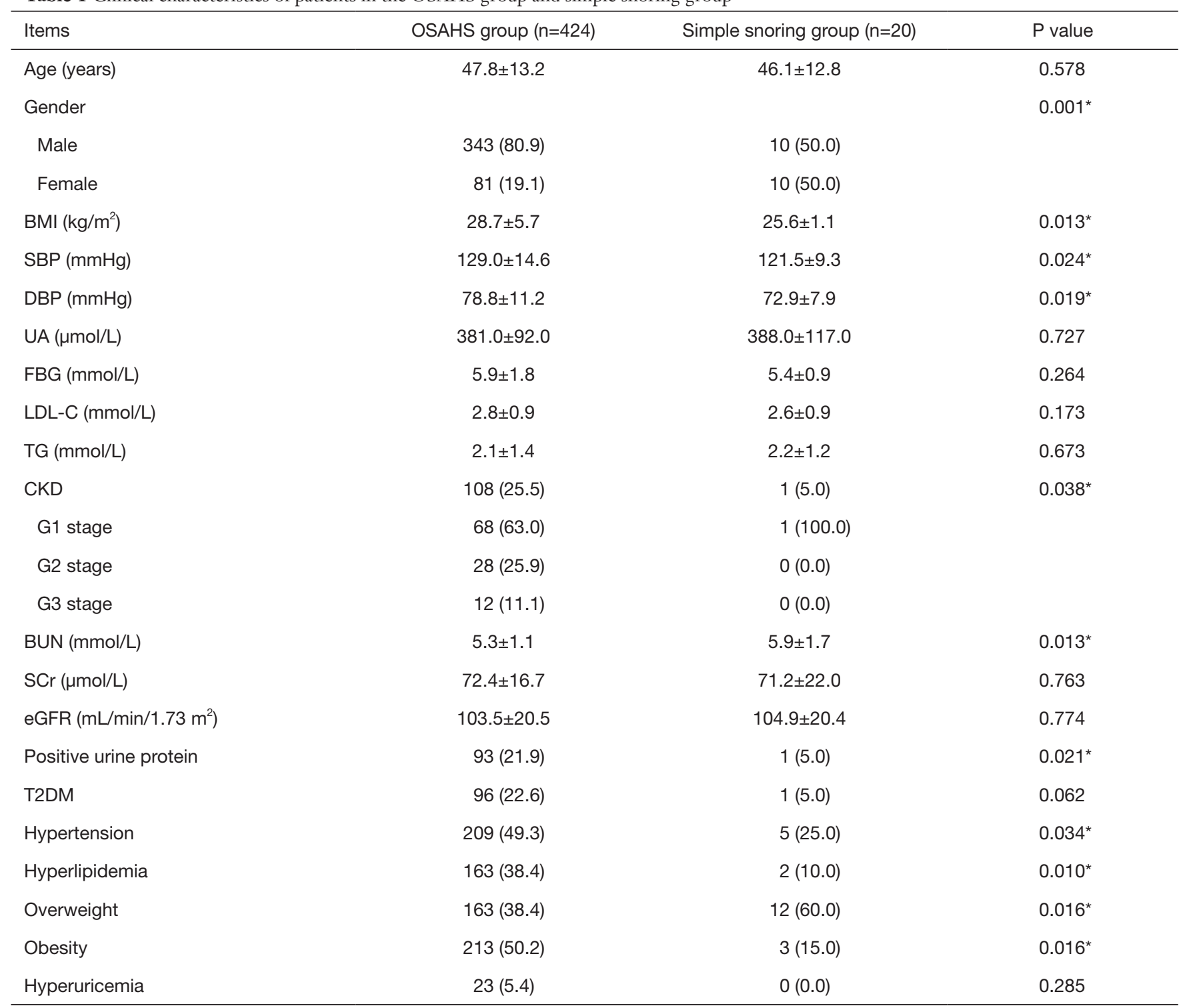

Data are expressed as mean \pm SD; counting data are represented as percentage. *, $\mathrm{P}<0.05$. OSAHS, obstructive sleep apnea-hypopnea syndrome; BMI, body mass index; SBP, systolic blood pressure; DBP, diastolic blood pressure; UA, uric acid; FBG, fasting blood glucose; LDL-C, low-density lipoprotein cholesterol; TG, triglyceride; CKD, chronic kidney disease; G1 chronic kidney disease stage 1; G2 chronic kidney disease stage 2; G3 chronic kidney disease stage 3; BUN, urea nitrogen; SCr, serum creatinine; eGFR, estimate glomerular filtration rate; T2DM, type 2 diabetes mellitus.

of CKD in the OSAHS group was 5.1 times higher than that in the control group. The CKD in the OSAHS patients was mainly in the stage G1-G2 and $11.1 \%$ of patients had stage G3 CKD. In the OSAHS group, 96 patients (22.6\%) comorbid with T2DM, 209 (49.3\%) with hypertension, 163 (38.4\%) with hyperlipidemia, $163(38.4 \%)$ with overweight, $213(50.2 \%)$ with obesity, and $23(5.4 \%)$ with hyperuric acid. The prevalence of CKD in the OSAHS patients was significantly higher than that in the simple snoring group $(\mathrm{P}=0.038)$. Meanwhile, there were statistically significant differences in the gender $(\mathrm{P}=0.001)$, metabolic related factors such as systolic blood pressure $(\mathrm{SBP})(\mathrm{P}=0.024)$, diastolic blood pressure $(\mathrm{DBP})(\mathrm{P}=0.019)$, obesity $(\mathrm{P}=0.016)$ and hyperlipidemia $(\mathrm{P}=0.010)$ between OSAHS and control group. The sample size of the simple snoring group was small and there might be selection bias. In the OSAHS 
Table 2 Clinical characteristics of CKD in the OSAHS patients with and without metabolic diseases

\begin{tabular}{|c|c|c|c|}
\hline Items & Metabolic disease group $(n=326)$ & Non-metabolic disease group $(n=98)$ & $P$ value \\
\hline Gender & & & 0.210 \\
\hline Male & 268 (82.2) & $75(76.5)$ & \\
\hline Female & $58(17.8)$ & $23(23.5)$ & \\
\hline eGFR $\left(\mathrm{mL} / \mathrm{min} / 1.73 \mathrm{~m}^{2}\right)$ & $101.8 \pm 22.1$ & $109.1 \pm 12.8$ & $0.002^{*}$ \\
\hline Positive urine protein & $79(24.2)$ & $14(14.3)$ & 0.263 \\
\hline $\mathrm{SCr}(\mu \mathrm{mol} / \mathrm{L})$ & $73.5 \pm 17.6$ & $68.4 \pm 12.4$ & $0.009^{\star}$ \\
\hline BUN (mmol/L) & $5.3 \pm 1.1$ & $5.1 \pm 1.1$ & 0.154 \\
\hline BMI $\left(\mathrm{kg} / \mathrm{m}^{2}\right)$ & $29.9 \pm 5.8$ & $24.7 \pm 2.6$ & $0.000^{*}$ \\
\hline $\mathrm{FBG}(\mathrm{mmol} / \mathrm{L})$ & $6.1 \pm 1.9$ & $5.2 \pm 1.0$ & $0.000^{*}$ \\
\hline GHB (\%) & $6.5 \pm 1.5$ & $5.8 \pm 1.4$ & $0.011^{*}$ \\
\hline LDL-C (mmol/L) & $2.8 \pm 0.9$ & $2.9 \pm 0.9$ & 0.788 \\
\hline TC (mmol/L) & $4.5 \pm 0.9$ & $4.7 \pm 1.0$ & 0.090 \\
\hline $\mathrm{TG}(\mathrm{mmol} / \mathrm{L})$ & $2.2 \pm 1.5$ & $2.0 \pm 1.2$ & 0.209 \\
\hline $\mathrm{UA}(\mu \mathrm{mol} / \mathrm{L})$ & $385.0 \pm 94.0$ & $366.0 \pm 79.0$ & 0.084 \\
\hline WBC $\left(\times 10^{9} / L\right)$ & $7.03 \pm 1.70$ & $6.97 \pm 6.09$ & 0.873 \\
\hline
\end{tabular}

Data are expressed as mean $\pm \mathrm{SD}$; counting data are represented as percentage. * $\mathrm{P}<0.05$. CKD, chronic kidney disease; OSAHS, obstructive sleep apnea-hypopnea syndrome; eGFR, estimate glomerular filtration rate; SCr, serum creatinine; BUN, urea nitrogen; SBP, systolic blood pressure; DBP, diastolic blood pressure; BMI, body mass index; FBG, fasting blood glucose; GHB, glycosylated hemoglobin; LDL-C, low-density lipoprotein cholesterol; TC, total cholesterol; TG, triglyceride; UA, uric acid; WBC, white blood cell; NE, neutrophilic granulocyte; PLT, blood platelet.

group, 40 patients matched with sex and age in the simple snoring group were selected $(2: 1)$. The prevalence of CKD in the OSAHS group was 5.5 times higher than that in the simple snoring group $(\mathrm{P}=0.040)$, and there were significant differences in the body mass index $(\mathrm{BMI})(\mathrm{P}=0.005)$, urea nitrogen $(\mathrm{P}=0.020)$ and urinary protein $(\mathrm{P}=0.033)$ between OSAHS group and simple snoring group $(2: 1)$. OSAHS patients had a high prevalence of CKD.

\section{CKD in OSAHS patients with metabolic diseases}

To investigate the influence of metabolic diseases on the CKD, 424 OSAHS patients were divided into metabolic disease group non-metabolic disease group. The metabolic diseases included obesity, hypertension, T2DM, hyperlipidemia and hyperuricemia. Three hundred and twenty-six OSAHS patients comorbid with metabolic diseases and 98 without metabolic diseases. The prevalence of CKD was $28.5 \%$ in OSAHS comorbid with metabolic disease, which was 1.9 times that in OSAHS alone ( $\mathrm{P}=0.008)$. OSAHS patients with metabolic diseases were susceptible to CKD (Table 2).

\section{eGFR related factors in OSAHS patients with metabolic diseases}

The OSAHS patients comorbid with metabolic diseases 
Table 3 eGFR related factors in the OSAHS patients without obesity

\begin{tabular}{lllllllll}
\hline eGFR & $\mathrm{AHI}$ & $\mathrm{LaSO}_{2}$ & ODI & BMI & SBP & FBG & LDL-C & UA \\
\hline$r$ & 0.140 & -0.254 & 0.134 & 0.037 & -0.274 & -0.178 & 0.138 \\
$\mathrm{P}$ & $0.042^{*}$ & $0.000^{*}$ & 0.052 & 0.598 & $0.000^{*}$ & $0.010^{*}$ & $0.046^{*}$ & 0.212 \\
\hline
\end{tabular}

*, P<0.05. eGFR, estimate glomerular filtration rate; OSAHS, obstructive sleep apnea-hypopnea syndrome; AHI, Apnea-Hypopnea Index; $\mathrm{LaSO}_{2}$, lowest blood oxygen saturation; ODI, oxygen desaturation index; BMI, body mass index; SBP, systolic blood pressure; FBG, fasting blood glucose; LDL-C, low-density lipoprotein cholesterol; UA, uric acid.

Table 4 eGFR related factors in OSAHS patients without hypertension

\begin{tabular}{lccccccccr}
\hline eGFR & $\mathrm{AHI}$ & $\mathrm{LaSO}_{2}$ & $\mathrm{ODI}$ & $\mathrm{BMI}$ & $\mathrm{SBP}$ & $\mathrm{DBP}$ & FBG & LDL-C & UA \\
\hline $\mathrm{r}$ & 0.184 & -0.141 & 0.172 & 0.169 & -0.039 & 0.068 & -0.072 & 0.040 & -0.036 \\
$\mathrm{P}$ & $0.007^{*}$ & $0.039^{*}$ & $0.012^{*}$ & $0.013^{*}$ & 0.574 & 0.318 & 0.292 & 0.564 & 0.599 \\
\hline
\end{tabular}

*, P<0.05. eGFR, estimate glomerular filtration rate; OSAHS, obstructive sleep apnea-hypopnea syndrome; AHI, Apnea-Hypopnea Index; $\mathrm{LaSO}_{2}$, lowest blood oxygen saturation; ODI, oxygen desaturation index; BMI, body mass index; SBP, systolic blood pressure; DBP, diastolic blood pressure; FBG, fasting blood glucose; LDL-C, low-density lipoprotein cholesterol; UA, uric acid.

Table 5 eGFR related factors in the OSAHS patients without T2DM

\begin{tabular}{lccccccccc}
\hline eGFR & $\mathrm{AHI}$ & $\mathrm{LaSO}_{2}$ & $\mathrm{ODI}$ & $\mathrm{BMI}$ & $\mathrm{SBP}$ & $\mathrm{DBP}$ & FBG & LDL-C & UA \\
\hline $\mathrm{r}$ & 0.218 & -0.160 & 0.181 & 0.097 & -0.115 & 0.006 & -0.008 & 0.052 & 0.023 \\
$\mathrm{P}$ & $0.000^{*}$ & $0.004^{*}$ & $0.001^{*}$ & 0.081 & $0.037^{*}$ & 0.907 & 0.884 & 0.349 & 0.684 \\
\hline
\end{tabular}

* $\mathrm{P}<0.05$. eGFR, estimate glomerular filtration rate; OSAHS, obstructive sleep apnea-hypopnea syndrome; AHI, Apnea-Hypopnea Index; $\mathrm{LaSO}_{2}$, lowest blood oxygen saturation; ODI, oxygen desaturation index; BMI, body mass index; SBP, systolic blood pressure; DBP, diastolic blood pressure; FBG, fasting blood glucose; LDL-C, low-density lipoprotein cholesterol; UA, uric acid.

had a significantly higher prevalence of CKD. Excluding each metabolic disease (obesity/hypertension/T2DM/ hyperlipidemia/hyperuricemia) one by one, the correlation between PSG parameters [AHI and lowest blood oxygen saturation $\left.\left(\mathrm{LaSO}_{2}\right)\right]$ and metabolic diseases was further studied in the OSAHS patients.

\section{eGFR related factors in OSAHS patients without obesity}

In 211 non-obese patients, the eGFR as a dependent variable, PSG parameters and metabolic risk factors as independent variables were integrated into the correlation analysis model (Table 3). Results showed eGFR was positively related to AHI $(\mathrm{r}=0.140, \mathrm{P}=0.042)$, ODI $(\mathrm{r}=0.134$, $\mathrm{P}=0.052), \mathrm{FBG}(\mathrm{r}=0.178, \mathrm{P}=0.010)$ and low-density lipoprotein cholesterol $(\mathrm{LDL}-\mathrm{C})(\mathrm{r}=0.138, \mathrm{P}=0.046)$ and negatively related to $\mathrm{LaSO}_{2}(\mathrm{r}=-0.254, \mathrm{P}<0.000)$ and SBP $(\mathrm{r}=-0.274, \mathrm{P}<0.000)$. After adjustment for obesity factors, eGFR increased with the AHI and ODI, and decreased with the $\mathrm{LaSO}_{2}$.

\section{eGFR related factors in OSAHS patients without hypertension}

In 215 OSAHS patients without hypertension, the eGFR as a dependent variable, PSG parameters and metabolic risk factors as independent variables were integrated into the correlation analysis model (Table 4). Results showed eGFR was positively related to AHI $(\mathrm{r}=0.184, \mathrm{P}=0.007)$, ODI $(r=0.172, \mathrm{P}=0.012)$ and $\mathrm{BMI}(\mathrm{r}=0.169, \mathrm{P}=0.013)$, and negatively related to $\mathrm{LaSO}_{2}(\mathrm{r}=-0.014, \mathrm{P}=0.039)$. After adjustment for hypertension, eGFR increased with the AHI, ODI and BMI, and decreased with the $\mathrm{LaSO}_{2}$.

\section{eGFR related factors in OSAHS patients without T2DM}

In 328 OSAHS patients without hypertension, the eGFR as a dependent variable, PSG parameters and metabolic risk factors as independent variables were integrated into the correlation analysis model (Table 5). Results showed eGFR was positively related to AHI $(r=0.218, \mathrm{P}<0.000)$ and ODI $(r=0.181, \mathrm{P}=0.001)$, and negatively related to $\mathrm{LaSO}_{2}$ 
Table 6 eGFR related factors in the OSAHS patients without hyperlipidemia

\begin{tabular}{lccccccccc}
\hline eGFR & $\mathrm{AHI}$ & $\mathrm{LaSO}_{2}$ & ODI & BMI & SBP & DBP & FBG & LDL-C & UA \\
\hline$r$ & 0.195 & -0.121 & 0.179 & 0.091 & -0.121 & 0.040 & -0.055 & 0.010 & -0.085 \\
$\mathrm{P}$ & $0.002^{*}$ & 0.050 & $0.004^{*}$ & 0.145 & 0.051 & 0.521 & 0.379 & 0.877 & 0.172 \\
\hline
\end{tabular}

*, P<0.05. eGFR, estimate glomerular filtration rate; OSAHS, obstructive sleep apnea-hypopnea syndrome; AHI, Apnea-Hypopnea Index; $\mathrm{LaSO}_{2}$, lowest blood oxygen saturation; ODI, oxygen desaturation index; BMI, body mass index; SBP, systolic blood pressure; DBP, diastolic blood pressure; FBG, fasting blood glucose; LDL-C, low-density lipoprotein cholesterol; UA, uric acid.

Table 7 eGFR related factors in the OSAHS patients without hyperuricemia

\begin{tabular}{lccccccccc}
\hline eGFR & $\mathrm{AHI}$ & $\mathrm{LaSO}_{2}$ & $\mathrm{ODI}$ & $\mathrm{BMI}$ & $\mathrm{SBP}$ & $\mathrm{DBP}$ & FBG & LDL-C & UA \\
\hline $\mathrm{r}$ & 0.170 & -0.158 & 0.149 & 0.052 & -0.189 & -0.029 & -0.126 & 0.092 & -0.018 \\
$\mathrm{P}$ & $0.001^{*}$ & $0.002^{*}$ & $0.003^{*}$ & 0.300 & $0.000^{*}$ & 0.565 & $0.012^{*}$ & 0.065 & 0.726 \\
\hline
\end{tabular}

*, P<0.05. eGFR, estimate glomerular filtration rate; OSAHS, obstructive sleep apnea-hypopnea syndrome; AHI, Apnea-Hypopnea Index; $\mathrm{LaSO}_{2}$, lowest blood oxygen saturation; ODI, oxygen desaturation index; BMI, body mass index; SBP, systolic blood pressure; DBP, diastolic blood pressure; FBG, fasting blood glucose; LDL-C, low-density lipoprotein cholesterol; UA, uric acid.

$(\mathrm{r}=-0.160, \mathrm{P}=0.004)$ and $\mathrm{SBP}(\mathrm{r}=-0.115, \mathrm{P}=0.037)$. After adjustment for diabetes, eGFR increased with the AHI and ODI, and decreased with $\mathrm{LaSO}_{2}$ and SBP.

\section{eGFR related factors in OSAHS patients without} hyperlipidemia

In 261 OSAHS patients without hyperlipidemia, the eGFR as a dependent variable, PSG parameters and metabolic risk factors as independent variables were integrated into the correlation analysis model (Table 6). eGFR was positively related to AHI $(r=0.218, P=0.000)$ and ODI $(r=0.181$, $\mathrm{P}=0.001)$, and negatively related to $\mathrm{LaSO}_{2}(\mathrm{r}=-0.160$, $\mathrm{P}=0.004)$ and $\mathrm{SBP}(\mathrm{r}=-0.115, \mathrm{P}=0.037)$. After adjustment for hyperlipidemia, eGFR increased with AHI and ODI, and decreased with $\mathrm{LaSO}_{2}$.

\section{eGFR related factors in OSAHS patients without} hyperuricemia

In 401 OSAHS patients without hyperuricemia, the eGFR as a dependent variable, the PSG parameters and metabolic risk factors as independent variables were integrated into the correlation analysis model (Table 7). eGFR was positively related to $\mathrm{AHI}(\mathrm{r}=0.170, \mathrm{P}=0.001)$ and ODI $(r=0.149, \mathrm{P}=0.003)$ and negatively related to $\mathrm{LaSO}_{2}$ $(\mathrm{r}=-0.158, \mathrm{P}=0.002)$, SBP $(\mathrm{r}=-0.189, \mathrm{P}<0.000)$ and FBG $(\mathrm{r}=-0.126, \mathrm{P}=0.012)$. After adjustment for hyperuricemia, eGFR increased with AHI and ODI, and decreased with $\mathrm{LaSO}_{2}, \mathrm{SBP}$ and FBG.
Risk factors of CKD in OSAHS patients by univariate analysis

The above findings suggest that not only AHI, $\mathrm{LaSO}_{2}$, ODI were significantly related to eGFR, but also BMI, SBP, FBG and LDL-C did. According to the AHI, OSAHS patients with or without metabolic disease were subdivided into mild to moderate group (AHI $=5-30$ times/h) and severe group ( $\mathrm{AHI}>30$ times $/ \mathrm{h}$ ). The prevalence of $\mathrm{CKD}$, urinary protein, eGFR and metabolic diseases were compared between two groups. Results are shown in Tables 8,9. There was no significant difference between mild to moderate OSAHS group and severe OSAHS group in patients without metabolic diseases as shown in univariate analysis. These results indicate that OSAHS alone and its severity are not risk factors for CKD. Metabolic diseases may become confounding factors.

\section{Multivariate analysis of risk factors for CKD in OSAHS patients with metabolic diseases}

\section{Multiple linear regression analysis of eGFR risk} factors in OSAHS patients with metabolic diseases

Based on the above results, further adjustment for confounding factors was performed in the multiple linear regression analysis, aiming to investigate the relationship of eGFR and OSAHS with metabolic diseases. The correlation between OSAHS and eGFR was assessed in 326 OSAHS patients with metabolic diseases. eGFR as a 
Table 8 Clinical characteristics of OSAHS patients without metabolic diseases

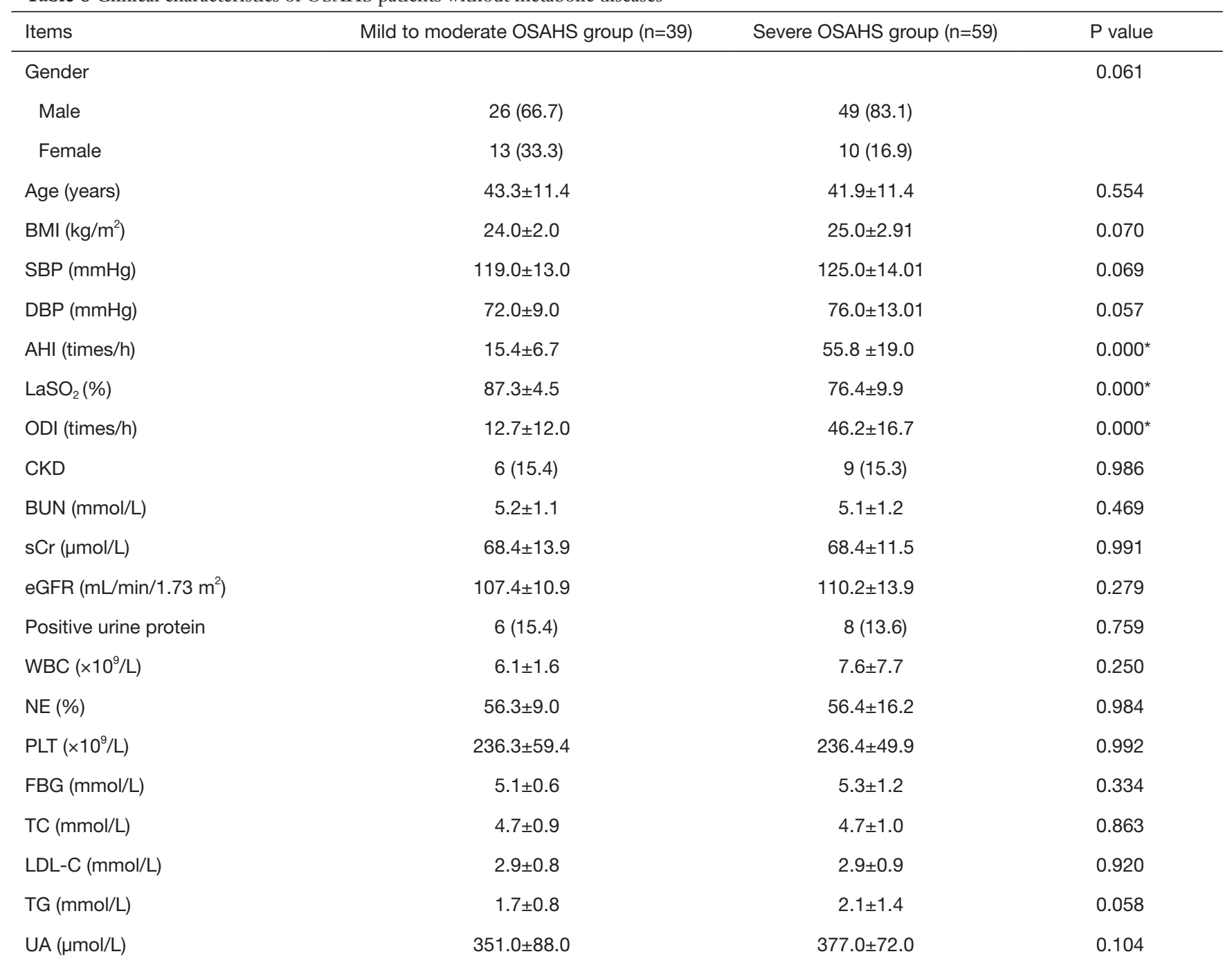

Data are expressed as mean \pm SD; counting data are represented as percentage. ${ }^{*}, \mathrm{P}<0.05$. OSAHS, obstructive sleep apnea-hypopnea syndrome; BMI, body mass index; SBP, systolic blood pressure; DBP, diastolic blood pressure; AHI, Apnea-Hypopnea Index; LaSO lowest blood oxygen saturation; ODI, oxygen desaturation index; CKD, chronic kidney disease; BUN, urea nitrogen; SCr, serum creatinine; eGFR, estimate glomerular filtration rate; WBC, white blood cell; NE, neutrophilic granulocyte; PLT, blood platelet; PLT, blood platelet; FBG, fasting blood glucose; TC, total cholesterol; LDL-C, low-density lipoprotein cholesterol; TG, triglyceride; UA, uric acid.

dependent variable, and age, metabolic related factors and PSG parameters as independent variables were integrated into the multiple linear regression model with stepwise method. The results are shown in Table 10. AHI $(\mathrm{P}=0.022)$, $\mathrm{LaSO}_{2}(\mathrm{P}=0.012)$, ODI $(\mathrm{P}=0.021)$ and SBP $(\mathrm{P}=0.031)$ had significant effects on the eGFR.

\section{Independent risk factors of CKD in OSAHS patients}

The independent risk factors of CKD were assessed in 424 OSAHS patients by using binary Logistic multivariate regression analysis after adjustment for confounding factors. CKD served as a dependent variable. Age, gender, and metabolic diseases (obesity, hypertension, T2DM, hyperlipidemia and hyperuricemia) served as independent variables in the binary Logistic regression model. The results are shown in Table 11. OSAHS with T2DM was an independent risk factor for $\mathrm{CKD}[\mathrm{P}=0.045$, odds ratio $(\mathrm{OR})=0.566,95 \%$ confidence interval (CI): 0.324-0.989]. The prevalence of CKD in OSAHS patients with T2DM combined with other 1-4 metabolic diseases was $25.0 \%$, 
Table 9 Clinical characteristics of OSAHS patients with metabolic diseases

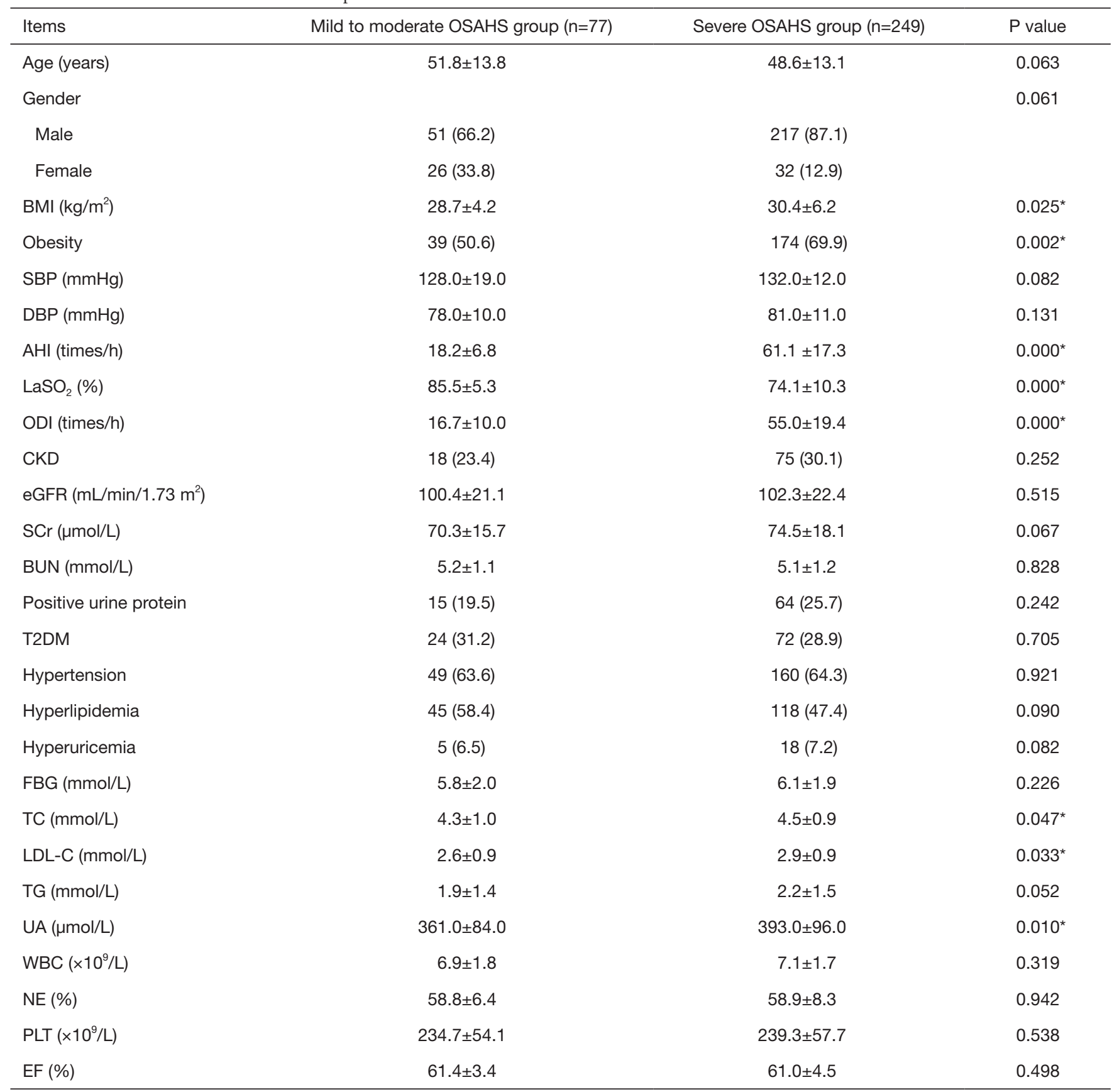

Data are expressed as mean $\pm \mathrm{SD}$; counting data are represented as percentage. ${ }^{*}, \mathrm{P}<0.05$. OSAHS, obstructive sleep apnea-hypopnea syndrome; BMI, body mass index; SBP, systolic blood pressure; DBP, diastolic blood pressure; $\mathrm{AHI}$, Apnea-Hypopnea Index; LaSO ${ }_{2}$, lowest blood oxygen saturation; ODI, oxygen desaturation index; CKD, chronic kidney disease; BUN, urea nitrogen; SCr, serum creatinine; eGFR, estimate glomerular filtration rate; T2DM, type 2 diabetes mellitus; FBG, fasting blood glucose; TC, total cholesterol; LDL-C, lowdensity lipoprotein cholesterol; TG, triglyceride; UA, uric acid; WBC, white blood cell; NE, neutrophilic granulocyte; PLT, blood platelet; PLT, blood platelet; EF, ejection fraction. 
Table 10 Multiple linear regression analysis of eGFR risk factors in OSAHS patients with metabolic diseases

\begin{tabular}{lccc}
\hline Independent variables & Beta & $t$ & P \\
\hline AHI (times/h) & 0.104 & 2.301 & $0.022^{*}$ \\
LaSO $_{2}(\%)$ & -0.115 & -2.541 & $0.012^{*}$ \\
ODI (times/h) & 0.104 & 2.314 & $0.021^{*}$ \\
BMI (kg/m²) & -0.009 & -0.191 & 0.849 \\
SBP $(\mathrm{mmHg})$ & -0.097 & -2.166 & $0.031^{*}$ \\
DBP $(\mathrm{mmHg})$ & -0.054 & -1.206 & 0.229 \\
LDL-C (mmol/L) & -0.042 & -0.912 & 0.363 \\
TG (mmol/L) & -0.35 & -0.768 & 0.443 \\
FBG (mmol/L) & -0.059 & -1.322 & 0.187 \\
\hline
\end{tabular}

* $\mathrm{P}<0.05$. eGFR, estimate glomerular filtration rate; OSAHS, obstructive sleep apnea-hypopnea syndrome; AHI, Apnea-Hypopnea Index; $\mathrm{LaSO}_{2}$, lowest blood oxygen saturation; ODI, oxygen desaturation index; BMI, body mass index; SBP, systolic blood pressure; DBP, diastolic blood pressure; LDL-C, low-density lipoprotein cholesterol; TG, triglyceride; FBG, fasting blood glucose.

Table 11 Independent risk factors analysis of CKD in OSAHS patients (Logistic regression)

\begin{tabular}{lcccc}
\hline Items & $\mathrm{B}$ & $\mathrm{P}$ & $\mathrm{OR}$ & \multicolumn{1}{c}{$95 \% \mathrm{Cl}$} \\
\hline Age & -0.005 & 0.636 & 0.995 & $0.976-1.015$ \\
Gender & -0.606 & $0.038^{\star}$ & 0.546 & $0.308-0.967$ \\
Obesity & -0.450 & 0.067 & 0.638 & $0.394-1.032$ \\
Hypertension & 0.205 & 0.450 & 1.227 & $0.721-2.089$ \\
T2DM & -0.568 & $0.045^{*}$ & 0.566 & $0.324-0.989$ \\
Hyperlipidemia & -0.497 & 0.064 & 0.609 & $0.360-1.028$ \\
Hyperuricemia & -0.465 & 0.320 & 0.628 & $0.252-1.569$ \\
\hline
\end{tabular}

*, P<0.05. CKD, chronic kidney disease; OSAHS, obstructive sleep apnea-hypopnea syndrome; T2DM, type 2 diabetes mellitus; OR, odds ratio; $\mathrm{Cl}$, confidence interval.

$34.4 \%, 43.2 \%$ and $66.7 \%$, respectively. The prevalence of $\mathrm{CKD}$ increased with the number of metabolic diseases.

\section{Discussion}

The prevalence of CKD is about $10.8 \%$ in China (10). Metabolic diseases such as diabetes and obesity may accelerate the progression of CKD. With the increasing awareness of sleep-related symptoms such as daytime sleepiness and snoring, OSAHS has been timely diagnosed and treated. At the same time, CKD is gradually observed in those patients. The evidence shows that OSAHS is associated with CKD, but there is certain selection bias in available studies. The correlation between OSAHS and $\mathrm{CKD}$ is still inconclusive as there are many confounding risks factors. In the present retrospective study, metabolic diseases and CKD were investigated in the OSAHS patients, the correlation between OSAHS and CKD was assessed, and the influence of metabolic diseases was discussed, which may provide evidence on the early prevention, diagnosis and treatment of OSAHS and active control and/or delay of complications (including CKD).

Our results showed OSAHS patients had a higher prevalence of $\mathrm{CKD}$, and the prevalence of CKD increased with the aggravation of OSAHS, which was more common in males. In addition, the prevalence of CKD in OSAHS comorbid with metabolic diseases was significantly higher (28.5\% vs. $15.3 \%)$. OSAHS comorbid with metabolic diseases may be a risk factor for CKD. Moreover, our findings indicate a significant correlation between OSAHS 
and CKD, but OSAHS may not be an independent risk factor for CKD. Other metabolic factors may also affect the occurrence and development of CKD. After successively excluding metabolic diseases such as obesity and hypertension, eGFR correlated with not only PSG parameters, but also metabolic related factors.

Iseki et al. (11) investigated 1,624 patients with sleepbreathing disorder, and their results showed the prevalence of CKD was 3 times higher than that in the control group (30.5\% vs. 9.1\%). A follow-up cohort study of large population in Taiwan showed that the risk ratio of CKD in 4,319 patients with obstructive sleep apnea (OSA) was 1.37 (95\% CI: $1.05-1.77$ ) within 10 years and CKD occurred 2.5 months earlier than that in healthy population after excluding hypertension and T2DM. Chou et al. (12) also found a significant correlation between the severity of OSA and the renal injury in non-diabetic and hypertensive patients. However, Canales et al. (13) failed to find out renal dysfunction in patients with sleep-disordered breathing. Lee et al. (14) showed that OSA was a risk factor for CKD only in the presence of metabolic syndrome. Although there is correlation between OSAHS and CKD to a certain extent, metabolic diseases such as obesity, hypertension, T2DM, hyperlipidemia and hyperuricemia may also affect this association. Of note, metabolic diseases and other interfering factors are not excluded in many studies. Our study confirmed that OSAHS comorbid with metabolic diseases might be a risk factor for CKD, and OSAHS itself might not be an independent risk factor for CKD, which is consistent with previous findings (14), but more studies are needed to confirm this finding.

In the present study, the eGFR in OSAHS patients with metabolic diseases, regardless of obesity, T2DM, hypertension, hyperlipidemia and hyperuricemia, was positively related to AHI, and negatively related to ODI and $\mathrm{LaSO}_{2}$. This might be explained as the hypoxia induced renal dysfunction, oxidative stress, increased autonomic nerve excitability, and increased compensation of glomerular filtration function after rein-angiotensin-aldosterone system (RAAS) activation, which is consistent with the theory of glomerular hyperfiltration. When the dilation of afferent arterioles increases the blood flow to the kidney, the glomerular capillary pressure gradually increases, and the long-term renal hypertension results in glomerular sclerosis and increases protein load. In patients with sleep-disordered breathing, nocturnal hypoxemia may stimulate chronic inflammation and fibrosis in the renal interstitial, causing CKD. Chou et al. (12) recruited 40 habitual snoring subjects without diabetes, hypertension and other basic diseases. AHI, ratio of eGFR and urinary albumin/creatinine (ACR) were monitored. The results showed AHI was positively related to ACR and eGFR. However, most studies have concluded that eGFR decreases gradually with the aggravation of OSAHS or AHI. Ahmed et al. (15) followed up 858 patients with no history of CKD and with suspected sleep apnea for an average 2.1 years, and results showed nocturnal hypoxia in $44 \%$ of the patients and eGFR decrement of $>4 \mathrm{~mL} / \mathrm{min} / 1.73 \mathrm{~m}^{2}$ (equivalent to normal $2-4$ times) in $5.7 \%$ of patients after adjustment for the respiratory disturbance index, age, BMI, diabetes, heart failure, showing a significant correlation, OR $=2.89$ (95\% CI: $1.25-6.67)$. It is suggested that night hypoxia is independently associated with the decline in renal function. The abnormal increase of eGFR in OSAHS patients at the early stage of kidney disease is a compensation for renal filtration. More attention should be paid to this pathology, and the influence of metabolic diseases cannot be ignored. In this study, long-term follow-up of OSAHS patients is still required to investigate the trend of renal function, and regular monitoring of renal function and urinary protein is helpful for the early identification of CKD in OSAHS patients.

In this study, the prevalence of CKD in OSAHS patients with metabolic diseases was nearly 2 times higher than that in the OSAHS patients without metabolic diseases, suggesting that metabolic related factors may affect the occurrence of CKD. Multiple factors regression analysis showed eGFR had significant effect on AHI, $\mathrm{LaSO}_{2}$, ODI and SBP in OSAHS patients with metabolic diseases. OSAHS comorbid with diabetes was an independent risk factor for CKD, and significantly higher prevalence of CKD. Chou et al. (12) recruited 40 habitual snoring subjects without diabetes and hypertension and results showed that OSAHS was an independent risk factor for CKD after adjustment for diabetes, hypertension, age and other factors. Lee et al. (16) reached the same conclusion after adjustment for the gender, age and comorbidity diseases. Kanbay et al. (17) drew the same conclusion. Due to the confused factors such as diabetes, hypertension and BMI, OSAHS has proven to be an independent risk factor for CKD. Lee et al. (16) investigated the risk factors of CKD in patients with OSA, with metabolic syndrome as the main variable and their results showed only OSA with metabolic syndrome was an independent risk factor for CKD.

The main pathophysiology of OSAHS is chronic intermittent hypoxia at night, recurrent hypoxia, 
hypercapnia and sleep disruption. In this study, OSAHS patients with metabolic diseases were further assessed, and results showed OSAHS alone was not an independent risk factor for CKD. Diabetes played an important role in the relationship between OSAHS and CKD. Duo to the existence of diabetes, the incidence of CKD gradually increased with the accumulation of other metabolic diseases. Liyori et al. (18) investigated thin mice exposed to intermittent hypoxia and found that liver glycogen output did not decrease, while insulin sensitivity and skeletal muscle utilization of blood glucose decreased, confirming that intermittent hypoxia is an independent factor of insulin resistance after excluding obesity. The mechanism by which OSAHS can cause and aggravate glucose metabolism disorders is mainly due to the high sympathetic nervous activation in the OSAHS patients. Intermittent hypoxia during sleep induces chronic systemic inflammatory responses, and inflammatory mediators lead to impaired insulin signaling and abnormal glucose tolerance. Sleep deprivation can directly cause glucose metabolism disorder.

Therefore, OSAHS comorbid with diabetes is very important for the occurrence of CKD and may accelerate the procession of CKD. In OSAHS patients comorbid with diabetes, accumulation of other metabolic diseases promotes renal damage. More studies are warranted to confirm our findings in the future.

\section{Conclusions}

The prevalence of CKD in OSAHS patients is relatively high. OSAHS comorbid with diabetes rather than OSAHS alone is an independent risk factor for CKD. The prevalence of CKD increases with the number of comorbidities of metabolic diseases.

\section{Limitations}

This study was a single-center study, and subjects were inpatients from sleep centers. Most of them were diagnosed with severe OSAHS. Also, it was a retrospective study and the urinary protein content was only qualitatively analyzed. The sample size is small in the simple snoring group due to the lack of hospitalization data.

\section{Acknowledgments}

Funding: None.

\section{Footnote}

Conflicts of Interest: All authors have completed the ICMJE uniform disclosure form (available at http://dx.doi. org/10.21037/apm.2020.03.21). The authors have no conflicts of interest to declare.

Etbical Statement: The authors are accountable for all aspects of the work in ensuring that questions related to the accuracy or integrity of any part of the work are appropriately investigated and resolved. The study was approved by Beijing Tsinghua Changgung Hospital (No. 20119-0-01) and written informed consent was obtained from all patients.

Open Access Statement: This is an Open Access article distributed in accordance with the Creative Commons Attribution-NonCommercial-NoDerivs 4.0 International License (CC BY-NC-ND 4.0), which permits the noncommercial replication and distribution of the article with the strict proviso that no changes or edits are made and the original work is properly cited (including links to both the formal publication through the relevant DOI and the license). See: https://creativecommons.org/licenses/by-nc-nd/4.0/.

\section{References}

1. Kapur VK, Auckley DH, Chowdhuri S, et al. Clinical Practice Guideline for Diagnostic Testing for Adult Obstructive Sleep Apnea: An American Academy of Sleep Medicine Clinical Practice Guideline. J Clin Sleep Med 2017;13:479-504.

2. Young T, Skatrud J, Peppard PE. Risk factors for obstructive sleep apnea in adults. JAMA 2004;291:2013-6.

3. Jennum P, Riha RL. Epidemiology of sleep apnoea/ hypopnoea syndrome and sleep-disordered breathing. Eur Respir J 2009;33:907-14.

4. Young T, Finn L, Austin D, et al. Menopausal status and sleep-disordered breathing in the Wisconsin Sleep Cohort Study. Am J Respir Crit Care Med 2003;167:1181-5.

5. Quintana-Gallego E, Carmona-Bernal C, Capote F, et al. Gender differences in obstructive sleep apnea syndrome: a clinical study of 1166 patients. Respir Med 2004;98:984-9.

6. Gottlieb DJ, Yenokyan G, Newman AB, et al. Prospective study of obstructive sleep apnea and incident coronary heart disease and heart failure: the sleep heart health study. Circulation 2010;122:352-60.

7. Hanly PJ, Ahmed SB. Sleep apnea and the kidney: is 
sleep apnea a risk factor for chronic kidney disease? Chest 2014;146:1114-22.

8. Stevens PE, Levin A. Evaluation and management of chronic kidney disease: synopsis of the kidney disease: improving global outcomes 2012 clinical practice guideline. Ann Intern Med 2013;158:825-30.

9. GBD 2016 Disease and Injury Incidence and Prevalence Collaborators. Global, regional, and national incidence, prevalence, and years lived with disability for 328 diseases and injuries for 195 countries, 1990-2016: a systematic analysis for the Global Burden of Disease Study 2016. Lancet 2017;390:1211-59.

10. Zhang L, Wang F, Wang L, et al. Prevalence of chronic kidney disease in China: a cross-sectional survey. Lancet 2012;379:815-22.

11. Iseki K, Tohyama K, Matsumoto T, et al. High Prevalence of chronic kidney disease among patients with sleep related breathing disorder (SRBD). Hypertens Res 2008;31:249-55.

12. Chou YT, Lee PH, Yang CT, et al. Obstructive sleep apnea: a stand-alone risk factor for chronic kidney disease. Nephrol Dial Transplant 2011;26:2244-50.

Cite this article as: Hui M, Li Y, Ye J, Zhuang Z, Wang W. Obstructive sleep apnea-hypopnea syndrome (OSAHS) comorbid with diabetes rather than OSAHS alone serves an independent risk factor for chronic kidney disease (CKD). Ann Palliat Med 2020;9(3):858-869. doi: 10.21037/apm.2020.03.21
13. Canales MT, Lui LY, Taylor BC, et al. Renal function and sleep-disordered breathing in older men. Nephrol Dial Transplant 2008;23:3908-14.

14. Lee YJ, Jang HR, Huh W, et al. Independent Contributions of Obstructive Sleep Apnea and the Metabolic Syndrome to the Risk of Chronic Kidney Disease. J Clin Sleep Med 2017;13:1145-52.

15. Ahmed SB, Ronksley PE, Hemmelgarn BR, et al. Nocturnal hypoxia and loss of kidney function. PLoS One 2011;6:e19029.

16. Lee J, Turin TC, Nicholl DD, et al. Predictors of successful completion of diagnostic home sleep testing in patients with chronic kidney disease. Sleep Breath 2015;19:669-75.

17. Kanbay A, Buyukoglan H, Ozdogan N, et al. Obstructive sleep apnea syndrome is related to the progression of chronic kidney disease. Int Urol Nephrol 2012;44:535-9.

18. Iiyori $\mathrm{N}$, Alonso LC, Li J, et al. Intermittent hypoxia causes insulin resistance in lean mice independent of autonomic activity. Am J Respir Crit Care Med 2007; 175:851-7. 